\title{
A simplified modal expansion formalism adapted to the optical design of resonant cavity enhanced photodetectors using metallic gratings
}

\author{
Maximilien Billet, Emilien Peytavit \\ IEMN (Institute of Electronics, Microelectronics and Nanotechnology) Univ. Lille, CNRS, \\ Centrale Lille, ISEN, Univ. Valenciennes, UMR 8520 - IEMN, F-59652 Villeneuve d'Ascq, \\ France
}

\begin{abstract}
In this communication, we present a method to model the light absorption in a resonant photoconductor based on a photoconductive layer sandwiched between a metallic grating in front side and a metallic backside mirror. We started from a modal expansion formalism previously developed in order to model the transmission and the reflection of an infinite array of holes and we added the effect of a stack of homogeneous layers ended by a metallic mirror. It is finally shown that the electromagnetic response of the structure calculated with this numerical method is in good agreement with a more rigorous method such as the rigorous coupled wave analysis, whereas it requires a much lower computing power.
\end{abstract}

Keywords: Ultrafast Photodetector, Modal expansion analysis, Diffraction Gratings

2010 MSC: 00-01, 99-00

\section{Introduction}

Ultrafast photodetectors are key devices for the generation and the detection of $\mathrm{THz}$ waves based on the laser and optoelectronics technologies [1],[2]. As far as now, the devices which present suitable properties for these applications,

Email address: emilien.peytavit@iemn.univ-lille1.fr (Emilien Peytavit) 
5 i.e. a $\mathrm{THz}$ frequency bandwidth and a high saturation photocurrent, are unitravelling carrier photodiodes [3] (UTC-PD) pumped by $1550 \mathrm{~nm}$ lasers and ultrafast photoconductors based on photoconductive materials presenting subpicosecond carrier life time. This second class of devices is dominated by $\mathrm{THz}$ photoconductors using GaAs epitaxial layers grown at low temperature $\left(200^{\circ}\right.$ 10 C ) [4] and pumped by 780-nm-lasers [5]. Last years, it has been shown that their properties could be greatly improved by using resonant optical cavities in which metallic layers serve in the same time as bias electrodes and mirrors of the cavity [6, [7]. Concerning the THz photoconductors, the optical cavity allows for reducing the thickness of the absorption layer while keeping constant the quantum efficiency (defined here as the ratio of the number of charge carrier generated in the device to the number of incident photons by unit of time) resulting in an increase of the photoresponse. This concept can also be adapted to the UTC-PD in order to reduce the thickness of the p-doped absorbing layer, and then improve the frequency bandwidth without loss in quantum efficiency.

20 The front mirror of the optical cavity is semi-transparent and consisted of a thin $(20 \mathrm{~nm})$ gold layer in the first attempts[6], however this solution suffers from some drawbacks such as high optical losses and high sheet electrical resistivities. In a second step, front mirrors consisting of a one dimensional array of slits has been considered, allowing for a better transmission and a lower effective sheet electrical resistivity [8]. In this work, we present a simplified modal expansion formalism (SMEF) based on the work of De Leon-Perez and co-workers [9], 10], in order to design such an optical cavity which needs a very low computing power as compared with more standard methods such as the rigorous coupled wave method.The SMEF is similar to a coupled mode analysis [11, but with some simplification coming from the use of waveguide modes in the slits patterned in the metallic sheet. Here, in a first part, we will remind the method as presented by De Leon-Perez and coworkers with the addition of some missing steps. In a second part we will present the extension the additional steps allowing for modeling model not only a metallic grating, but a also Fabry-Pérot cavity based 35 on such a grating. 


\section{Scattering of light by an array of metallic holes}

In this first part, we are going to remind the main steps of the SMEF method as presented in Ref. [9] allowing for the calculation of the electromagnetic response of an infinite two-dimensional array to an incident plane wave. The array is 40 assumed to be in the $x-y$ plane and has a finite thickness $h$, as we can see in Figure 1 The lattice parameters are $L_{x}$ and $L_{y}$. The analysis is performed by using the CGS system and only the transverse components of the electric field $\boldsymbol{E}$ and magnetic field $\boldsymbol{H}$, which are used in the boundary conditions at the interface between different media, are taking into account. The dirac notation is used,

45 such that $\left\langle\boldsymbol{r}_{\|} \mid \boldsymbol{E}\right\rangle=\boldsymbol{E}\left(\boldsymbol{r}_{\|}\right)=\left(E_{x}\left(\boldsymbol{r}_{\|}\right), E_{y}\left(\boldsymbol{r}_{\|}\right)\right)^{t}$ where $t$ stand for transposition and $\boldsymbol{r}_{\|}=(x, y)$. Regions I and III are dielectric semi-spaces characterized by the real dielectric constant $\epsilon_{1}$ and $\epsilon_{3}$, respectively. Region II represents the corrugated metal film with a wavelength-dependent dielectric function $\epsilon_{m}$. We assume that the system is illuminated by an incident wave coming from region I. We expand the EM fields on the eigenmodes of each region, and match them at the boundaries. In region I, the fields are expanded into an infinite set of plane waves with parallel wavevector $\boldsymbol{k}_{\|}=\left(k_{x}, k_{y}\right)$ and polarization $\sigma$. The incident radiation is labeled by the superscript 0 . The fields can be written in terms of

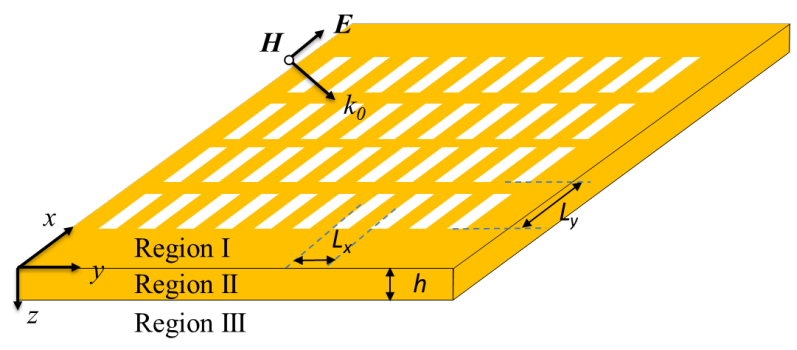

Figure 1: Schematic of a two dimensional array of holes perforated on a metal film of thickness $h$ illuminated by p-polarized radiation. For the case of s-polarized incident radiation, vectors $\boldsymbol{E}$ and $\boldsymbol{H}$ are interchanged 
reflection amplitude $\rho_{\boldsymbol{k} \| \sigma}$ as

$$
\begin{aligned}
\left|\boldsymbol{E}^{\mathrm{I}}\right\rangle & =e^{\mathrm{i} k_{z}^{0} z}\left|\boldsymbol{k}_{\|}^{0} \sigma^{0}\right\rangle+\sum_{\boldsymbol{k} \sigma} \rho_{\boldsymbol{k} \| \sigma} e^{-\mathrm{i} k_{z} z}\left|\boldsymbol{k}_{\|} \sigma\right\rangle \\
\left|-\boldsymbol{u}_{\boldsymbol{z}} \times \boldsymbol{H}^{\mathrm{I}}\right\rangle & =Y_{\boldsymbol{k}_{\|}^{0} \sigma^{0}} e^{\mathrm{i} k_{z}^{0} z}\left|\boldsymbol{k}_{\|}^{0} \sigma^{0}\right\rangle-\sum_{\boldsymbol{k}_{\|} \sigma} Y_{\boldsymbol{k}_{\|} \sigma} \rho_{\boldsymbol{k} \| \sigma} e^{-\mathrm{i} k_{z} z}\left|\boldsymbol{k}_{\|} \sigma\right\rangle
\end{aligned}
$$

where $\boldsymbol{u}_{\boldsymbol{z}}$ is the unitary vector along the $z$-direction and the summation runs over parallel wavevectors such as $\boldsymbol{k}_{\|}=\boldsymbol{k}_{\|}^{0}+\boldsymbol{K}_{\boldsymbol{R}}, \boldsymbol{K}_{\boldsymbol{R}}$ being a vector of the reciprocal lattice. The real space representation of the plane waves is given by

$$
\left\langle r_{\|} \mid \boldsymbol{k}_{\|} \sigma\right\rangle=\frac{\exp \left(\mathrm{i} \boldsymbol{k}_{\|} \cdot r\right)}{k_{\|} \sqrt{L_{x} L_{y}}} \begin{cases}\left(k_{x}, k_{y}\right)^{t}, & \text { p-polarization } \\ \left(-k_{y}, k_{x}\right)^{t}, & \text { s-polarization }\end{cases}
$$

where we assume a $\left.\| \boldsymbol{k}_{\|} \sigma\right\rangle$ independent normalization $\left\langle\boldsymbol{k}_{\|} \sigma \mid \boldsymbol{k}_{\|} \sigma\right\rangle=1$ The electric and magnetic field are related through the admittance $Y_{\boldsymbol{k} \| p}=k_{\omega} \epsilon_{1} / k_{z}$ and $Y_{\boldsymbol{k} \| s}=k_{z} / k_{\omega}$ where $k_{\omega}=2 \pi / \lambda_{0}\left(\lambda_{0}\right.$ is the free space wavelength of the incident radiation, and $k_{\|}^{2}+k_{z}^{2}=\epsilon_{1} k_{\omega}^{2}$ (see Appendix A for further details).

\subsection{Expansion of the fields in the 3 regions}

${ }_{60}$ We have already seen in equation (1) the expansion on a basis of plane waves in the region I. In this first part, we assume that the Region III $(z \geq h)$ is semi-infinite, so the EM fields can also be expanded in plane waves and expressed in terms of transmission amplitudes $t_{\boldsymbol{k} \| \sigma}$ :

$$
\begin{aligned}
\left|\boldsymbol{E}^{\mathrm{III}}\right\rangle & =\sum_{\boldsymbol{k} \sigma} t_{\boldsymbol{k} \| \sigma} e^{\mathrm{i} k_{z}^{\prime}(z-h)}\left|\boldsymbol{k}_{\|} \sigma\right\rangle \\
\left|-\boldsymbol{u}_{\boldsymbol{z}} \times \boldsymbol{H}^{\mathrm{III}}\right\rangle & =\sum_{\boldsymbol{k}_{\|} \sigma} Y_{\boldsymbol{k}_{\|}}^{\prime} t_{\boldsymbol{k} \| \sigma} e^{\mathrm{i} k_{z}^{\prime}(z-h)}\left|\boldsymbol{k}_{\|} \sigma\right\rangle
\end{aligned}
$$

Now, we are going to expand the EM fields in Region II on a waveguide modes basis corresponding to the geometry of the holes. Noting $|\alpha\rangle$ a certain waveguide mode, and $C_{\alpha}$ and $D_{\alpha}$ the expansion coefficient, we can write:

$$
\begin{aligned}
\left|\boldsymbol{E}^{\mathrm{II}}\right\rangle & =\sum_{\alpha}\left(C_{\alpha} e^{\mathrm{i} k_{\alpha z} z}+D_{\alpha} e^{-\mathrm{i} k_{\alpha z} z}\right)|\alpha\rangle \\
\left|-\boldsymbol{u}_{\boldsymbol{z}} \times \boldsymbol{H}^{\mathrm{II}}\right\rangle & =\sum_{\alpha} Y_{\alpha}\left(C_{\alpha} e^{\mathrm{i} k_{\alpha z} z}-D_{\alpha} e^{-\mathrm{i} k_{\alpha z} z}\right)|\alpha\rangle
\end{aligned}
$$

where $Y_{\alpha}=k_{\omega} \epsilon_{2} / k_{\alpha z}$ and $Y_{\alpha}=k_{\alpha z} / k_{\omega}$ for TE and TM modes respectively. 


\subsection{Application of the boundary conditions at each region interface}

Following Ref. [9, we use the crude approximation in order to take into account the real properties of the metal sheet forming the grating.It establishes the following relation between the tangential components of the EM fields at the horizontal interface: $\boldsymbol{E}=z_{s} \boldsymbol{H} \times \boldsymbol{u}_{\boldsymbol{n}}$, where $\boldsymbol{u}_{\boldsymbol{n}}$ is the vector normal to the surface and pointing to the interioir of the metal and $z_{s}=1 / \sqrt{\epsilon_{M}}$. This continuity equation must be satisfied at each interface. In Region I $u_{n}=u_{z}$ and the continuity equation gives :

$$
\begin{array}{rccc}
z_{s} H_{x} & 0 & z_{s} H_{y} & E_{x} \\
z_{s} \boldsymbol{H} \times \boldsymbol{u}_{\boldsymbol{z}}= & z_{s} H_{y} \times 0=-z_{s} H_{x}=z_{s} \boldsymbol{Y}_{\boldsymbol{k} \| \boldsymbol{\sigma}} \cdot E_{y} \\
z_{s} H_{z} & 1 & 0 & 0
\end{array}
$$

70 2.2.1. Continuity of the Electric Field in $\mathrm{z}=0$

In $z=0$ and with the dirac notations, it gives:

$$
\begin{gathered}
\left|\boldsymbol{E}^{\mathrm{I}}\right\rangle=z_{s} Y_{\boldsymbol{k} \| \sigma}\left|\boldsymbol{E}^{\mathrm{I}}\right\rangle \\
\Longleftrightarrow \\
\left|\boldsymbol{k}_{\|}^{0} \sigma^{0}\right\rangle+\sum_{\boldsymbol{k} \sigma} \rho_{\boldsymbol{k} \| \sigma}\left|\boldsymbol{k}_{\|} \sigma\right\rangle=z_{s} Y_{\boldsymbol{k}_{\|}^{0} \sigma^{0}}\left|\boldsymbol{k}_{\|}^{0} \sigma^{0}\right\rangle-\sum_{\boldsymbol{k}_{\|} \sigma} z_{s} Y_{\boldsymbol{k}_{\|} \sigma} \rho_{\boldsymbol{k} \| \sigma}\left|\boldsymbol{k}_{\|} \sigma\right\rangle
\end{gathered}
$$

Now, if we project on the plane waves basis $\left\langle\boldsymbol{k}_{\|} \sigma\right|$, we obtain:

$$
\delta_{\boldsymbol{k} \|, \boldsymbol{k}_{\|}^{0}} \delta_{\sigma, \sigma^{0}}+\rho_{\boldsymbol{k} \| \sigma}=z_{s} Y_{\boldsymbol{k}_{\|}^{0} \sigma^{0}} \delta_{\boldsymbol{k} \|, \boldsymbol{k}_{\|}^{0}} \delta_{\sigma, \sigma^{0}}-z_{s} Y_{\boldsymbol{k}_{\|} \sigma} \rho_{\boldsymbol{k} \| \sigma}
$$

In Region II, in $z=0^{+}$, with also $u_{n}=u_{z}$, it gives

$$
\sum_{\alpha}\left(C_{\alpha}+D_{\alpha}\right)|\alpha\rangle=\sum_{\alpha} z_{s} Y_{\alpha}\left(C_{\alpha}-D_{\alpha}\right)|\alpha\rangle
$$

And once projected on the $\left\langle\boldsymbol{k}_{\|} \sigma\right|$ basis:

$$
\sum_{\alpha}\left(C_{\alpha}+D_{\alpha}\right)\left\langle\boldsymbol{k}_{\|} \sigma \mid \alpha\right\rangle=\sum_{\alpha} z_{s} Y_{\alpha}\left(C_{\alpha}-D_{\alpha}\right)\left\langle\boldsymbol{k}_{\|} \sigma \mid \alpha\right\rangle
$$


If we have the continuity of the Electric Field in $z=0$, we must equalize both terms of equation 10 and equation 12 . Then it gives:

$$
\begin{aligned}
\sum_{\alpha}\left(C_{\alpha}+D_{\alpha}\left\langle\boldsymbol{k}_{\|} \sigma \mid \alpha\right\rangle\right. & =\delta_{\boldsymbol{k} \|, \boldsymbol{k}_{\|}^{0}} \delta_{\sigma, \sigma^{0}}+\rho_{\boldsymbol{k} \| \sigma} \\
\sum_{\alpha} z_{s} Y_{\alpha}\left(C_{\alpha}-D_{\alpha}\right)\left\langle\boldsymbol{k}_{\|} \sigma \mid \alpha\right\rangle & =z_{s} Y_{\boldsymbol{k}_{\|}^{0} \sigma^{0}} \delta_{\boldsymbol{k} \|, \boldsymbol{k}^{0}} \delta_{\sigma, \sigma^{0}}-z_{s} Y_{\boldsymbol{k}_{\|} \sigma} \rho_{\boldsymbol{k} \| \sigma}
\end{aligned}
$$

(13)-14 gives:

$$
\begin{gathered}
\sum_{\alpha}\left\langle\boldsymbol{k}_{\|} \sigma \mid \alpha\right\rangle E_{\alpha}=\delta_{\boldsymbol{k} \|, \boldsymbol{k}_{\|}^{0}} \delta_{\sigma, \sigma^{0}}\left(1-z_{s} Y_{\boldsymbol{k}_{\|} \sigma}\right)+\rho_{\boldsymbol{k} \| \sigma}\left(1+z_{s} Y_{\boldsymbol{k}_{\|} \sigma}\right) \\
\Longleftrightarrow \\
\boldsymbol{\rho}_{\boldsymbol{k} \| \boldsymbol{\sigma}}=\frac{1}{\boldsymbol{f}_{\boldsymbol{k}_{\|} \boldsymbol{\sigma}}^{+}} \sum_{\boldsymbol{\alpha}}\left\langle\boldsymbol{k}_{\|} \boldsymbol{\sigma} \mid \boldsymbol{\alpha}\right\rangle \boldsymbol{E}_{\boldsymbol{\alpha}}-\frac{\boldsymbol{f}_{\boldsymbol{k}_{\|} \boldsymbol{\sigma}}^{-}}{\boldsymbol{f}_{\boldsymbol{k}_{\|} \boldsymbol{\sigma}}^{+}} \boldsymbol{\delta}_{\boldsymbol{k}_{\|}, \boldsymbol{k}_{\|}^{0}} \boldsymbol{\delta}_{\boldsymbol{\sigma}, \boldsymbol{\sigma}^{\mathrm{o}}}
\end{gathered}
$$

where $E_{\alpha}=C_{\alpha} f_{\alpha}^{-}+D_{\alpha} f_{\alpha}^{+}$and $f_{\alpha}^{ \pm}=1 \pm z_{s} Y_{\alpha}$.

\subsubsection{Continuity of the Electric Field in $z=h$}

In $z=h^{-}, u_{n}=-u_{z}$ and we have:

$$
\sum_{\alpha}\left(C_{\alpha} e^{\mathrm{i} k_{\alpha z} h}+D_{\alpha} e^{-\mathrm{i} k_{\alpha z} h}\right)|\alpha\rangle=-\sum_{\alpha} z_{s} Y_{\alpha}\left(C_{\alpha} e^{\mathrm{i} k_{\alpha z} h}-D_{\alpha} e^{-\mathrm{i} k_{\alpha z} h}\right)|\alpha\rangle
$$

In $z=h^{+}$,

$$
\sum_{\boldsymbol{k}_{\|} \sigma} t_{\boldsymbol{k} \| \sigma}\left|\boldsymbol{k}_{\|} \sigma\right\rangle=-\sum_{\boldsymbol{k}_{\|} \sigma} z_{s} Y_{\boldsymbol{k}_{\|} \sigma}^{\prime} t_{\boldsymbol{k} \| \sigma}\left|\boldsymbol{k}_{\|} \sigma\right\rangle
$$

Once projected on the $\left.\left\langle\boldsymbol{k}_{\|} \sigma\right|, 18-17\right)$ gives:

$t_{\boldsymbol{k} \| \sigma}-\sum_{\alpha}\left(C_{\alpha} e^{\mathrm{i} k_{\alpha z} h}+D_{\alpha} e^{-\mathrm{i} k_{\alpha z} h}\right)\left\langle\boldsymbol{k}_{\|} \sigma \mid \alpha\right\rangle=-z_{s} Y_{\boldsymbol{k}_{\|} \sigma}^{\prime} t_{\boldsymbol{k} \| \sigma}+\sum_{\alpha} z_{s} Y_{\alpha}\left(C_{\alpha} e^{\mathrm{i} k_{\alpha z} h}-D_{\alpha} e^{-\mathrm{i} k_{\alpha z} h}\right)\left\langle\boldsymbol{k}_{\|} \sigma \mid \alpha\right\rangle$

$$
\Longleftrightarrow
$$

$$
t_{k \| \sigma}=-\frac{1}{f_{k_{\|} \sigma}^{\prime+}} \sum_{\alpha}\left\langle k_{\|} \sigma \mid \alpha\right\rangle E_{\alpha}^{\prime}
$$

${ }_{75}$ where $E_{\alpha}^{\prime}=-C_{\alpha} f_{\alpha}^{+} e^{\mathrm{i} k_{\alpha z} h}-D_{\alpha} f_{\alpha}^{-} e^{-\mathrm{i} k_{\alpha z} h}$. 


\subsubsection{Continuity of the Magnetic Field}

Let us now impose the continuity of the tangential component of the magnetic field which has to be fulfill only in the slits. This is done by projecting the fields on the $|\alpha\rangle$ basis. In $z=0$, we obtain:

$$
Y_{\boldsymbol{k}_{\|}^{0} \sigma^{0}}\left|\boldsymbol{k}_{\|}^{0} \sigma^{0}\right\rangle-\sum_{\boldsymbol{k}_{\|} \sigma} Y_{\boldsymbol{k}_{\|} \sigma} \rho_{\boldsymbol{k} \| \sigma}\left|\boldsymbol{k}_{\|} \sigma\right\rangle=\sum_{\alpha} Y_{\alpha}\left(C_{\alpha}-D_{\alpha}\right)|\alpha\rangle
$$

and once projected in the $|\alpha\rangle$, it becomes:

$$
Y_{\boldsymbol{k}_{\|}^{0} \sigma^{0}}\left\langle\alpha \mid \boldsymbol{k}_{\|}^{0} \sigma^{0}\right\rangle-\sum_{\boldsymbol{k}_{\|} \sigma} Y_{\boldsymbol{k}_{\|} \sigma} \rho_{\boldsymbol{k} \| \sigma}\left\langle\alpha \mid \boldsymbol{k}_{\|} \sigma\right\rangle=Y_{\alpha}\left(C_{\alpha}-D_{\alpha}\right)
$$

In $z=h$, once projected on $|\alpha\rangle$ we obtain:

$$
\sum_{\boldsymbol{k}_{\|} \sigma} Y_{\boldsymbol{k}_{\|} \sigma}^{\prime} t_{\boldsymbol{k} \| \sigma}\left\langle\alpha \mid \boldsymbol{k}_{\|} \sigma\right\rangle=Y_{\alpha}\left(C_{\alpha} e^{\mathrm{i} k_{\alpha z} h}-D_{\alpha} e^{-\mathrm{i} k_{\alpha z} h}\right)
$$

If we put the expressions of $\rho_{k \| \sigma}$ and $t_{k \| \sigma}$ given in equations (16) and 20] in theses expressions, we obtain for equation 22 :

$$
\begin{gathered}
Y_{\boldsymbol{k}_{\|}^{0} \sigma^{0}}\left\langle\alpha \mid \boldsymbol{k}_{\|}^{0} \sigma^{0}\right\rangle-\sum_{\boldsymbol{k}_{\|} \sigma} Y_{\boldsymbol{k}_{\|} \sigma}\left(\frac{1}{f_{\boldsymbol{k}_{\|} \sigma}^{+}} \sum_{\beta}\left\langle\boldsymbol{k}_{\|} \sigma \mid \beta\right\rangle E_{\beta}-\frac{f_{\boldsymbol{k}_{\|} \sigma}^{-} \sigma}{f_{\boldsymbol{k}_{\|} \sigma}^{+}} \delta_{\boldsymbol{k}_{\|}, \boldsymbol{k}_{\|}^{0}} \delta_{\sigma, \sigma^{0}}\right)\left\langle\alpha \mid \boldsymbol{k}_{\|} \sigma\right\rangle=Y_{\alpha}\left(C_{\alpha}-D_{\alpha}\right) \\
\Longleftrightarrow \\
\left\langle\alpha \mid \boldsymbol{k}_{\|}^{0} \sigma^{0}\right\rangle Y_{\boldsymbol{k}_{\|}^{0} \sigma^{0}}\left(1+\frac{f_{\boldsymbol{k}_{\|} \sigma}^{-}}{f_{\boldsymbol{k}_{\|} \sigma}^{+}}\right)-\sum_{\beta} \sum_{\boldsymbol{k}_{\|} \sigma} \frac{Y_{\boldsymbol{k}_{\|} \sigma}}{f_{\boldsymbol{k}_{\|} \sigma}^{+}}\left\langle\boldsymbol{k}_{\|} \sigma \mid \beta\right\rangle\left\langle\alpha \mid \boldsymbol{k}_{\|} \sigma\right\rangle E_{\beta}=Y_{\alpha}\left(C_{\alpha}-D_{\alpha}\right) \\
\Longleftrightarrow \\
\text { where } G_{\alpha \beta}=\sum_{\boldsymbol{k}_{\|} \sigma} \frac{Y_{\boldsymbol{k}_{\|} \sigma}}{f_{\boldsymbol{k}_{\|} \sigma}^{+}}\left\langle\alpha \mid \boldsymbol{k}_{\|} \sigma\right\rangle\left\langle\boldsymbol{k}_{\|} \sigma \mid \beta\right\rangle \text { and } I_{\alpha}=2 \frac{Y_{\boldsymbol{k}_{\|}^{0} \sigma^{0}}^{+}}{f_{\boldsymbol{k}_{\|}^{0} \sigma}^{+}}\left\langle\alpha \mid \boldsymbol{k}_{\|}^{0} \sigma^{0}\right\rangle .
\end{gathered}
$$


Now, let's show that $Y_{\alpha}\left(C_{\alpha}-D_{\alpha}\right)=-\Sigma_{\alpha} E_{\alpha}-G_{\alpha}^{V} E_{\alpha}^{\prime}$ where

$$
\begin{aligned}
\Sigma_{\alpha} & =Y_{\alpha} \frac{f_{\alpha}^{+} e^{\mathrm{i} k_{\alpha z} h}+f_{\alpha}^{-} e^{-\mathrm{i} k_{\alpha z} h}}{f_{\alpha}^{+2} e^{\mathrm{i} k_{\alpha z} h}-f_{\alpha}^{-2} e^{-\mathrm{i} k_{\alpha z} h}} \\
G_{\alpha}^{V} & =\frac{2 Y_{\alpha}}{f_{\alpha}^{+2} e^{\mathrm{i} k_{\alpha z} h}-f_{\alpha}^{-2} e^{-\mathrm{i} k_{\alpha z} h}} \\
-\Sigma_{\alpha} E_{\alpha}-G_{\alpha}^{V} E_{\alpha}^{\prime} & =-Y_{\alpha} \frac{C_{\alpha} e^{\mathrm{i} k_{\alpha z} h}\left(f_{\alpha}^{+} f_{\alpha}^{-}-2 f_{\alpha}^{+}\right)+C_{\alpha} e^{-\mathrm{i} k_{\alpha z} h} f_{\alpha}^{-2}}{f_{\alpha}^{+2} e^{\mathrm{i} k_{\alpha z} h}-f_{\alpha}^{-2} e^{-\mathrm{i} k_{\alpha z} h}} \\
- & Y_{\alpha} \frac{D_{\alpha} e^{-\mathrm{i} k_{\alpha z} h}\left(f_{\alpha}^{+} f_{\alpha}^{-}-2 f_{\alpha}^{-}\right)+D_{\alpha} e^{\mathrm{i} k_{\alpha z} h} f_{\alpha}^{+2}}{f_{\alpha}^{+2} e^{\mathrm{i} k_{\alpha z} h}-f_{\alpha}^{-2} e^{-\mathrm{i} k_{\alpha z} h}} \\
-\Sigma_{\alpha} E_{\alpha}-G_{\alpha}^{V} E_{\alpha}^{\prime} & =-Y_{\alpha} \frac{C_{\alpha} e^{-\mathrm{i} k_{\alpha z} h} f_{\alpha}^{-2}-C_{\alpha} f_{\alpha}^{2+} e^{\mathrm{i} k_{\alpha z} h}}{f_{\alpha}^{+2} e^{\mathrm{i} k_{\alpha z} h}-f_{\alpha}^{-2} e^{-\mathrm{i} k_{\alpha z} h}} \\
- & Y_{\alpha} \frac{D_{\alpha} e^{\mathrm{i} k_{\alpha z} h} f_{\alpha}^{+2}-D_{\alpha} f_{\alpha}^{2-} e^{-\mathrm{i} k_{\alpha z} h}}{f_{\alpha}^{+2} e^{\mathrm{i} k_{\alpha z} h}-f_{\alpha}^{-2} e^{-\mathrm{i} k_{\alpha z} h}} \\
& =Y_{\alpha}\left(C_{\alpha}-D_{\alpha}\right)
\end{aligned}
$$

We have used in the previous equation

$$
\begin{aligned}
f_{\alpha}^{+} f_{\alpha}^{-}-2 f_{\alpha}^{+} & =f_{\alpha}^{+}\left(f_{\alpha}^{-}-2\right) \\
& =\left(1+z_{s} Y_{\alpha}\right)\left(1-z_{s} Y_{\alpha}-2\right) \\
& =\left(1+z_{s} Y_{\alpha}\right)\left(-1-z_{s} Y_{\alpha}\right) \\
& =-f_{\alpha}^{2+}
\end{aligned}
$$

Concerning the second continuity equation (23), we obtain straightly:

$$
\sum_{\gamma \neq \nu} G_{\gamma \nu}^{\prime} E_{\nu}^{\prime}+G_{\gamma \gamma}^{\prime} E_{\gamma}^{\prime}=-Y_{\gamma}\left(C_{\gamma} e^{\mathrm{i} k_{\gamma z} h}-D_{\alpha} e^{-\mathrm{i} k_{\gamma z} h}\right)
$$

so It remains to prove that $\Sigma_{\gamma} E_{\gamma}^{\prime}+G_{\gamma}^{V} E_{\gamma}=-Y_{\gamma}\left(C_{\gamma} e^{\mathrm{i} k_{\gamma z} h}-D_{\gamma} e^{-\mathrm{i} k_{\gamma z} h}\right)$ 


$$
\begin{aligned}
\Sigma_{\alpha} E_{\alpha}^{\prime}+G_{\alpha}^{V} E_{\alpha} & =Y_{\alpha} \frac{\left(f_{\alpha}^{+} e^{\mathrm{i} k_{\alpha z} h}+f_{\alpha}^{-} e^{-\mathrm{i} k_{\alpha z} h}\right)\left(-C_{\alpha} f_{\alpha}^{+} e^{\mathrm{i} k_{\alpha z} h}-D_{\alpha} f_{\alpha}^{-} e^{-\mathrm{i} k_{\alpha z} h}\right)}{f_{\alpha}^{+2} e^{\mathrm{i} k_{\alpha z} h}-f_{\alpha}^{-2} e^{-\mathrm{i} k_{\alpha z} h}} \\
& +2 Y_{\alpha} \frac{C_{\alpha}+D_{\alpha}}{f_{\alpha}^{+2} e^{\mathrm{i} k_{\alpha z} h}-f_{\alpha}^{-2} e^{-\mathrm{i} k_{\alpha z} h}} \\
\Sigma_{\alpha} E_{\alpha}^{\prime}+G_{\alpha}^{V} E_{\alpha} & =Y_{\alpha} \frac{-C_{\alpha} f_{\alpha}^{2+} e^{2 \mathrm{i} k_{\alpha z} h}-D_{\alpha} f_{\alpha}^{2-} e^{-2 \mathrm{i} k_{\alpha z} h}+C_{\alpha}\left(2 f_{\alpha}^{-}-f_{\alpha}^{-} f_{\alpha}^{+}\right)+D_{\alpha}\left(2 f_{\alpha}^{+}-f_{\alpha}^{-} f_{\alpha}^{+}\right)}{f_{\alpha}^{+2} e^{\mathrm{i} k_{\alpha z} h}-f_{\alpha}^{-2} e^{-\mathrm{i} k_{\alpha z} h}} \\
& =Y_{\alpha} \frac{C_{\alpha}\left[f_{\alpha}^{-2}-f_{\alpha}^{+2} e^{2 \mathrm{i} k_{\alpha z} h}\right]+D_{\alpha}\left[f_{\alpha}^{+2}-f_{\alpha}^{-2} e^{-2 \mathrm{i} k_{\alpha z} h}\right]}{f_{\alpha}^{+2} e^{\mathrm{i} k_{\alpha z} h}-f_{\alpha}^{-2} e^{-\mathrm{i} k_{\alpha z} h}} \\
& =Y_{\alpha} \frac{C_{\alpha} e^{\mathrm{i} k_{\alpha z} h}\left[f_{\alpha}^{-2} e^{-\mathrm{i} k_{\alpha z} h}-f_{\alpha}^{+2} e^{\mathrm{i} k_{\alpha z} h}\right]+D_{\alpha} e^{-\mathrm{i} k_{\alpha z} h}\left[f_{\alpha}^{+2} e^{\mathrm{i} k_{\alpha z} h}-f_{\alpha}^{-2} e^{2 \mathrm{i} k_{\alpha z} h}\right]}{f_{\alpha}^{+2} e^{\mathrm{i} k_{\alpha z} h}-f_{\alpha}^{-2} e^{-\mathrm{i} k_{\alpha z} h}} \\
& =-Y_{\alpha}\left(C_{\alpha} e^{\mathrm{i} k_{\alpha z} h}-D_{\alpha} e^{-\mathrm{i} k_{\alpha z} h}\right)
\end{aligned}
$$

As in 9], we obtain eventually the set of coupled equations for $E_{\alpha}$ and $E_{\alpha}^{\prime}$ which are essentially the modal amplitudes of the electric field at the input and output interfaces of the holes, respectively:

$$
\begin{aligned}
\left(G_{\alpha \alpha}-\Sigma_{\alpha}\right) E_{\alpha}+\sum_{\beta \neq \alpha} G_{\alpha \beta} E_{\beta}-G_{\alpha}^{V} E_{\alpha}^{\prime} & =I_{\alpha} \\
\left(G_{\gamma \gamma}^{\prime}-\Sigma_{\gamma}\right) E_{\gamma}^{\prime}+\sum_{\nu \neq \gamma} G_{\gamma \nu}^{\prime} E_{\nu}^{\prime}-G_{\gamma}^{V} E_{\gamma} & =0
\end{aligned}
$$

Once $E_{\alpha}$ and $E_{\alpha}^{\prime}$ are known, all the other quantities can be calculated.

\section{Scattering on a 1D Periodic array of infinite slits}

\subsection{With only the $T M_{0}$ mode of the slit taken into account}

The problem is greatly simplified in the case of an array of infinite slits with a period $p=L_{x}$ and a width $a$ perpendicular to the incidence plane $(\boldsymbol{x}-\boldsymbol{z}$ plane, i.e. $\boldsymbol{k}_{\|}=k_{x} \boldsymbol{x}$ as seen in 2 of a p-polarized plane wave, if we consider only the

$T M_{0}$ in the slit, we obtain:

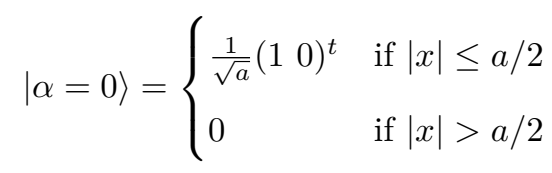




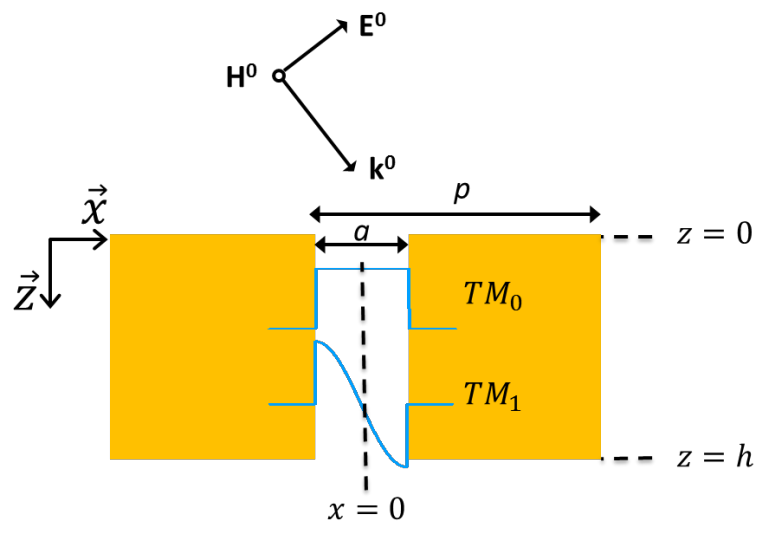

Figure 2: Cross section of one-dimensional array of infinite slits of width a perforated on a metal film of thickness $h$ illuminated by a p-polarized radiation

The normalization factor ensures that $\langle 0 \mid 0\rangle=\int_{x=-L_{x} / 2}^{x=L_{x} / 2} \frac{1}{a} d x=1$. Furthermore, by considering that the metal is perfect along the slit, we can assume that $k_{0 z}=k_{\omega}$.So, it is now possible to evaluate easily the overlap integral $G_{00}$ in the case of p-polarized incident wave because:

$$
\begin{aligned}
\left\langle 0 \mid k_{\|} \sigma\right\rangle & =(10)\left(k_{x} 0\right)^{t} \frac{1}{k_{x} \sqrt{a p}} \int_{x=-p / 2}^{x=p / 2} \exp \left(\mathrm{i} k_{x} x\right) d x \\
& =\frac{1}{\sqrt{a p}} \frac{\exp \left(\mathrm{i} k_{x} a / 2\right)-\exp \left(-\mathrm{i} k_{x} a / 2\right)}{\mathrm{i} k_{x}} \\
& =\sqrt{\frac{a}{p}} \frac{\sin \left(\frac{k_{x} a}{2}\right)}{\frac{k_{x} a}{2}}
\end{aligned}
$$

95 And then

$$
\begin{aligned}
G_{00} & =\sum_{\boldsymbol{k}_{\|} \sigma} \frac{Y_{\boldsymbol{k}_{\|} \sigma}}{f_{\boldsymbol{k}_{\|} \sigma}^{+}}\left|\left\langle 0 \mid \boldsymbol{k}_{\|} \sigma\right\rangle\right|^{2} \\
& =\sum_{\boldsymbol{k}_{\|} \sigma} \frac{Y_{\boldsymbol{k}_{\|} \sigma}}{f_{\boldsymbol{k}_{\|} \sigma}^{+}} \frac{a}{p} \frac{\sin ^{2}\left(\frac{k_{x} a}{2}\right)}{\left(\frac{k_{x} a}{2}\right)^{2}}
\end{aligned}
$$

Here the metallic sheet is assumed to be a perfect electric conductor allowing for an analytical expression of the overlap integration. Nevertheless it is possible to use a first order correction to improve the accuracy of the model [12] According the Bloch theorem, the sum runs over $\boldsymbol{k}_{\|}=k_{x} \boldsymbol{x}$ where $k_{x}=k_{x}^{0}+n \frac{2 \pi}{p}, n$ being 
an integer. The system of equations to be solved becomes:

$$
\begin{aligned}
& \left(G_{00}-\Sigma_{0}\right) E_{0}-G_{0}^{V} E_{0}^{\prime}=I_{0} \\
& \left(G_{00}^{\prime}-\Sigma_{0}\right) E_{0}^{\prime}-G_{0}^{V} E_{0}=0
\end{aligned}
$$

where

$$
\begin{aligned}
G_{00} & =\sum_{\boldsymbol{k}_{\|} \sigma} \frac{Y_{\boldsymbol{k}_{\|} \sigma}}{f_{\boldsymbol{k}_{\|} \sigma}^{+}} \frac{a}{p} \frac{\sin ^{2}\left(\frac{k_{x} a}{2}\right)}{\left(\frac{k_{x} a}{2}\right)^{2}} \\
I_{0} & =2 \sqrt{\frac{a}{p}} \frac{Y_{\boldsymbol{k}_{\|}^{0} \sigma^{0}}}{f_{\boldsymbol{k}_{\|}^{0} \sigma}^{+}} \frac{\sin \left(\frac{k_{x}^{0} a}{2}\right)}{\left(\frac{k_{x}^{0} a}{2}\right)} \\
\Sigma_{0} & =Y_{0} \frac{f_{0}^{+} e^{\mathrm{i} k_{0 z} h}+f_{0}^{-} e^{-\mathrm{i} k_{0 z} h}}{f_{0}^{+2} e^{\mathrm{i} k_{0 z} h}-f_{0}^{-2} e^{-\mathrm{i} k_{0 z} h}} \\
G_{0}^{V} & =\frac{2 Y_{0}}{f_{0}^{+2} e^{\mathrm{i} k_{0 z} h}-f_{0}^{-2} e^{-\mathrm{i} k_{0 z} h}}
\end{aligned}
$$

The solution of this set of equation is: $E_{0}^{\prime}=I_{0} G_{0}^{V} / D$ and $E_{0}=\left(G_{00}^{\prime}-\Sigma_{0}\right) / D$, where $D=\left(G_{00}-\Sigma_{0}\right)\left(G_{00}^{\prime}-\Sigma_{0}\right)-\left(G_{0}^{V}\right)^{2}$. Once $E_{0}^{\prime}$ and $E_{0}$ are obtained, we can calculate the transmission $t_{\boldsymbol{k}_{\|} p}$ and reflection coefficients $\rho_{\boldsymbol{k} \| p}$ f or each diffracted order and then the transmitted and reflected power are given by:

$$
\begin{aligned}
& T=\sum_{\boldsymbol{k} \| p}^{\text {propag }} Y_{\boldsymbol{k}_{\|} p}^{\prime}\left|t_{\boldsymbol{k} \| p}\right|^{2} \\
& R=\sum_{\boldsymbol{k}_{\| p}}^{\text {propag }} Y_{\boldsymbol{k}_{\| p}}\left|\rho_{\boldsymbol{k}_{\| p}}\right|^{2}
\end{aligned}
$$

propag indicates that only the propagative plane waves solutions should be taken into account.

\subsection{With the $T M_{0}$ and the $T M_{1}$ modes of the slit taken into account}

When the width of the slit becomes close to $\lambda / 2$, the second $T M_{1}$ mode in the slit has to be also taken into account. This mode is noted here $|\alpha=1\rangle$ and is defined as follows, if we consider perfect metal walls:

$$
|\alpha=1\rangle= \begin{cases}\frac{\sqrt{2}}{\sqrt{a}} \sin \left(\frac{\pi}{a} x\right)(10)^{t} & \text { if }|x| \leq a / 2 \\ 0 & \text { if }|x|>a / 2\end{cases}
$$


The normalization factor ensures that $\langle 1 \mid 1\rangle=\int_{x=-L_{x} / 2}^{x=L_{x} / 2} \frac{2}{a} \sin ^{2}\left(\frac{\pi}{a} x\right) d x=1$. Furthermore, $k_{1 z}=\sqrt{k_{\omega}^{2}-(\pi / a)^{2}}$.

So, it is now possible to evaluate $G_{11}$ in the case of p-polarized incident wave because:

$$
\begin{aligned}
\left\langle 1 \mid k_{\|} \sigma\right\rangle= & (10)\left(k_{x} 0\right)^{t} \frac{\sqrt{2}}{k_{x} \sqrt{a p}} \int_{x=-p / 2}^{x=p / 2} \sin \left(\frac{\pi}{a} x\right) \exp \left(\mathrm{i} k_{x} x\right) d x \\
= & \begin{cases}\mathrm{i} \cos \left(\frac{a k_{x}}{2}\right) \frac{(2 a)^{3 / 2}}{\left(\pi^{2}-k_{x}^{2} a^{2}\right) \sqrt{p}} & \text { if }\left|k_{x}\right| \neq \frac{\pi}{a} \\
\mathrm{i} \frac{\sqrt{2 \pi}}{\sqrt{p}\left|k_{x}\right|^{3 / 2}} & \text { if }\left|k_{x}\right|=\frac{\pi}{a}\end{cases}
\end{aligned}
$$

And then we can evaluate $G_{11}, G_{01}, G_{10}, G_{1}^{V}, \Sigma_{1}$ and resolve the system of equations given below in order to obtain $E_{0}, E_{1}, E_{0}^{\prime}$ and $E_{1}^{\prime}$ and then $T$ and $R$.

$$
\begin{aligned}
& \left(G_{00}-\Sigma_{0}\right) E_{0}+G_{01} E_{1}-G_{0}^{V} E_{0}^{\prime}=I_{0} \\
& \left(G_{11}-\Sigma_{1}\right) E_{1}+G_{10} E_{0}-G_{1}^{V} E_{1}^{\prime}=I_{1} \\
& \left(G_{00}^{\prime}-\Sigma_{0}\right) E_{0}^{\prime}+G_{01} E_{1}^{\prime}-G_{0}^{V} E_{0}=0 \\
& \left(G_{11}^{\prime}-\Sigma_{1}\right) E_{1}^{\prime}+G_{10} E_{0}^{\prime}-G_{1}^{V} E_{1}=0
\end{aligned}
$$

\section{Scattering on a 1D Periodic array of slits form- ing the top mirror of a Fabry-Perot cavity pho- todetector}

Now, we are interesting in the reflection and absorption of the system shown in Figure 3 where the periodic array of slits is backed by a stack of dielectric layers finished by a semi-infinite metallic layer serving as back-mirror of the cavity. We have to consider the waves reflected by the stacked of the layers and then the expression of the field in the region III (here we insure that $\epsilon_{3}$ defined in the case of a grid without cavity is equal to $\epsilon_{n}$ defined here). The field in the region III 


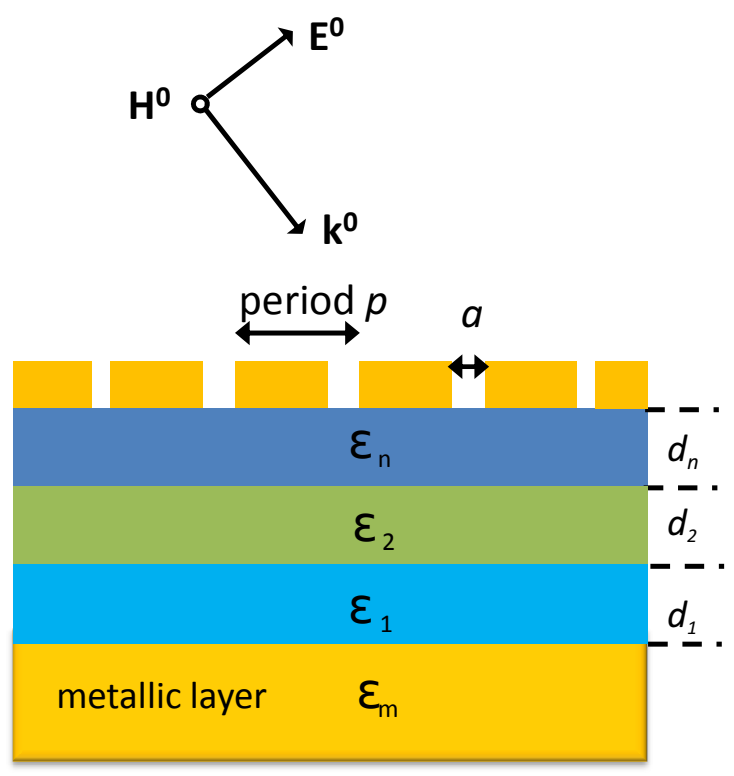

Figure 3: Schematic of an array of slits of width a perforated on a metal film of thickness $h$ on the top of a stack of dielectric layers of dielectric constants $\epsilon_{i}$ ended by a metallic mirror. 
4. SCATTERING ON A 1D PERIODIC ARRAY OF SLITS FORMING THE

TOP MIRROR OF A FABRY-PEROT CAVITY PHOTODETECTOR

becomes (see equation (3) expressed in terms of transmission amplitudes $t_{\boldsymbol{k} \| \sigma}$ :

$$
\begin{aligned}
\left|\boldsymbol{E}^{\mathrm{III}}\right\rangle & =\sum_{\boldsymbol{k} \sigma}\left(t_{\boldsymbol{k} \| \sigma} e^{\mathrm{i} k_{z}^{\prime}(z-h)}+\Pi_{\boldsymbol{k} \| \sigma} e^{-\mathrm{i} k_{z}^{\prime}(z-h)}\right)\left|\boldsymbol{k}_{\|} \sigma\right\rangle \\
\left|-\boldsymbol{u}_{\boldsymbol{z}} \times \boldsymbol{H}^{\mathrm{III}}\right\rangle & =\sum_{\boldsymbol{k}_{\|} \sigma} Y_{\boldsymbol{k}_{\|} \sigma}^{\prime}\left(t_{\boldsymbol{k} \| \sigma} e^{\mathrm{i} k_{z}^{\prime}(z-h)}-\Pi_{\boldsymbol{k} \| \sigma} e^{-\mathrm{i} k_{z}^{\prime}(z-h)}\right)\left|\boldsymbol{k}_{\|} \sigma\right\rangle
\end{aligned}
$$

\subsection{Continuity of the Electric Field in $z=h$}

The continuity equation of the Electric Field in $z=h$ (same method as in equation (17) and (18)) gives once projected on the $\left\langle\boldsymbol{k}_{\|} \sigma\right|$ :

In $z=h^{-}, u_{n}=-u_{z}$ and we have:

$$
\sum_{\alpha}\left(C_{\alpha} e^{\mathrm{i} k_{\alpha z} h}+D_{\alpha} e^{-\mathrm{i} k_{\alpha z} h}\right)|\alpha\rangle=-\sum_{\alpha} z_{s} Y_{\alpha}\left(C_{\alpha} e^{\mathrm{i} k_{\alpha z} h}-D_{\alpha} e^{-\mathrm{i} k_{\alpha z} h}\right)|\alpha\rangle
$$

In $z=h^{+}$,

$$
\sum_{\boldsymbol{k}_{\| \sigma}}\left(t_{\boldsymbol{k} \| \sigma}+\Pi_{\boldsymbol{k} \| \sigma}\right)\left|\boldsymbol{k}_{\|} \sigma\right\rangle=\sum_{\boldsymbol{k}_{\|} \sigma}-z_{s} Y_{\boldsymbol{k}_{\|} \sigma}^{\prime}\left(t_{\boldsymbol{k} \| \sigma}-\Pi_{\boldsymbol{k} \| \sigma}\right)\left|\boldsymbol{k}_{\|} \sigma\right\rangle
$$

Once projected on the $\left.\left\langle\boldsymbol{k}_{\|} \sigma\right|, 41\right]-40$ gives:

$$
\begin{array}{r}
\left(t_{\boldsymbol{k} \| \sigma}+\Pi_{\boldsymbol{k} \| \sigma}\right)-\sum_{\alpha}\left(C_{\alpha} e^{\mathrm{i} k_{\alpha z} h}+D_{\alpha} e^{-\mathrm{i} k_{\alpha z} h}\right)\left\langle\boldsymbol{k}_{\|} \sigma \mid \alpha\right\rangle= \\
-z_{s} Y_{\boldsymbol{k}_{\|} \sigma}^{\prime}\left(t_{\boldsymbol{k} \| \sigma}-\Pi_{\boldsymbol{k} \| \sigma}\right)+\sum_{\alpha} z_{s} Y_{\alpha}\left(C_{\alpha} e^{\mathrm{i} k_{\alpha z} h}-D_{\alpha} e^{-\mathrm{i} k_{\alpha z} h}\right)\left\langle\boldsymbol{k}_{\|} \sigma \mid \alpha\right\rangle \\
\Longleftrightarrow \\
\boldsymbol{f}_{\boldsymbol{k}_{\|} \boldsymbol{\sigma}}^{\prime+} \boldsymbol{t}_{\boldsymbol{k} \| \boldsymbol{\sigma}}+\boldsymbol{f}_{\boldsymbol{k}_{\|} \boldsymbol{\sigma}}^{\prime} \Pi_{\boldsymbol{k} \| \boldsymbol{\sigma}}=-\sum_{\boldsymbol{\alpha}}\left\langle\boldsymbol{k}_{\|} \boldsymbol{\sigma} \mid \boldsymbol{\alpha}\right\rangle \boldsymbol{E}_{\boldsymbol{\alpha}}^{\prime}
\end{array}
$$

The dielectrics layers below the scattering grid are assumed to be uniform and then the set of $\left|\boldsymbol{k}_{\|} \sigma\right\rangle$ is a subset of the orthogonal eigenmode basis (plane waves) which is independent of the layer. Therefore, as in a basic 1D uniform multilayer problem, no scattering occurs and we can calculate for each $\left|\boldsymbol{k}_{\|} \sigma\right\rangle$ the reflection coefficient $R_{\boldsymbol{k}_{\|} \sigma}$ brought by the cavity which is defined by:

$$
R_{\boldsymbol{k}_{\|} \sigma}=\frac{\Pi_{\boldsymbol{k}_{\|} \sigma}}{t_{\boldsymbol{k}_{\|} \sigma}}
$$




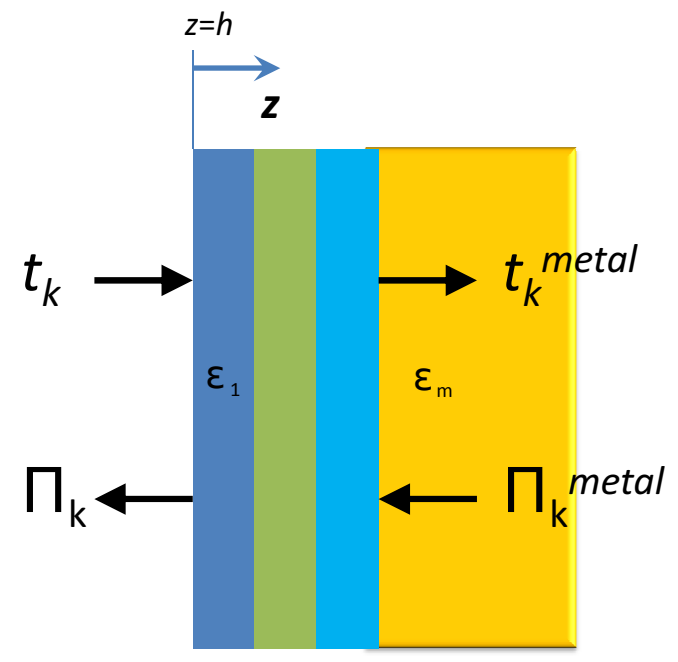

Figure 4: Forward and backward waves at the limits of the entire stack of layer

The reflection coefficient of a stack of uniform dielectric layers ended by a semi infinite layer (the metallic mirror here) is easily obtained by a transfer matrix method or a scattering matrix method (which is more robust, in terms of numerical stability, see [11] for example). We define the total S matrix as just below:

$$
\left|\begin{array}{l}
t_{\boldsymbol{k}_{\|} \sigma}^{\text {metal }} \\
\Pi_{\boldsymbol{k}_{\|} \sigma}
\end{array}\right|=\left|\begin{array}{ll}
S_{11} & S_{12} \\
S_{21} & S_{22}
\end{array}\right| \cdot\left|\begin{array}{c}
\Pi_{\boldsymbol{k}_{\|} \sigma}^{\text {metal }} \\
t_{\boldsymbol{k}_{\|} \sigma}
\end{array}\right|
$$

Once the total scattering matrix obtained (see fig. 4 for the definition of forward and backward waves ) which gives the linear dependency between the waves right after the metallic grid and at the beginning of the semi-infinite gold layer $R_{\boldsymbol{k}_{\|} \sigma}$ is easily calculated. Indeed, the backward wave in the metallic layer, $\Pi_{\boldsymbol{k}_{\|} \sigma}$ is null and then:

$$
R_{\boldsymbol{k}_{\|} \sigma}=S_{11}
$$

Now if we come back to eq. 42 we can obtain an equation similar to eq. 20 
4. SCATTERING ON A 1D PERIODIC ARRAY OF SLITS FORMING THE

TOP MIRROR OF A FABRY-PEROT CAVITY PHOTODETECTOR

found in the case of a grid without cavity but with a slight different factor.

$$
\begin{aligned}
-\sum_{\alpha}\left\langle\boldsymbol{k}_{\|} \sigma \mid \alpha\right\rangle E_{\alpha}^{\prime} & =f_{\boldsymbol{k}_{\|} \sigma}^{\prime} t_{\boldsymbol{k}_{\|} \sigma}+f_{\boldsymbol{k}_{\|} \sigma}^{\prime} t_{\boldsymbol{k}_{\|} \sigma} R_{\boldsymbol{k}_{\|} \sigma} \\
& =t_{\boldsymbol{k}_{\|} \sigma}\left(f_{\boldsymbol{k}_{\|} \sigma}^{\prime+}+f_{\boldsymbol{k}_{\|} \sigma}^{\prime-} R_{\boldsymbol{k}_{\|} \sigma}\right)
\end{aligned}
$$

which gives:

$$
t_{k_{\|} \sigma}=-\frac{1}{F_{k_{\|} \sigma}} \sum_{\alpha}\left\langle k_{\|} \sigma \mid \alpha\right\rangle E_{\alpha}^{\prime}
$$

where $F_{\boldsymbol{k}_{\|} \sigma}=f_{\boldsymbol{k}_{\|} \sigma}^{\prime}+f_{\boldsymbol{k}_{\|} \sigma}^{\prime-} R_{\boldsymbol{k}_{\|} \sigma}$

\subsection{Continuity of the Magnetic Field in $\mathrm{z}=\mathrm{h}$}

Now, it remains to use the second continuity equation at the backside interface where the problem is different from the grid without cavity. The continuity of the magnetic field gives after projection on $|\alpha\rangle$ :

$$
\begin{gathered}
\sum_{\boldsymbol{k}_{\|} \sigma} Y_{\boldsymbol{k}_{\|} \sigma}^{\prime}\left(t_{\boldsymbol{k}_{\|} \sigma}-\Pi_{\boldsymbol{k}_{\|} \sigma}\right)\left\langle\alpha \mid \boldsymbol{k}_{\|} \sigma\right\rangle=Y_{\alpha}\left(C_{\alpha} e^{\mathrm{i} k_{\alpha z} h}-D_{\alpha} e^{-\mathrm{i} k_{\alpha z} h}\right) \\
\Longleftrightarrow \\
\sum_{\boldsymbol{k}_{\|} \sigma} Y_{\boldsymbol{k}_{\|} \sigma}^{\prime} t_{\boldsymbol{k}_{\|} \sigma}\left(1-R_{\boldsymbol{k}_{\|} \sigma}\right)\left\langle\alpha \mid \boldsymbol{k}_{\|} \sigma\right\rangle=Y_{\alpha}\left(C_{\alpha} e^{\mathrm{i} k_{\alpha z} h}-D_{\alpha} e^{-\mathrm{i} k_{\alpha z} h}\right)
\end{gathered}
$$

If we put the expressions of $t_{k \| \sigma}$ given in equation 446 in theses expressions, we obtain:

$$
\begin{gathered}
\sum_{\boldsymbol{k}_{\|} \sigma} Y_{\boldsymbol{k}_{\|} \sigma}^{\prime} \frac{1}{F_{\boldsymbol{k}_{\|} \sigma}} \sum_{\beta}\left\langle\boldsymbol{k}_{\|} \sigma \mid \beta\right\rangle E_{\beta}^{\prime}\left(1-R_{\boldsymbol{k}_{\|} \sigma}\right)\left\langle\alpha \mid \boldsymbol{k}_{\|} \sigma\right\rangle=-Y_{\alpha}\left(C_{\alpha} e^{\mathrm{i} k_{\alpha z} h}-D_{\alpha} e^{-\mathrm{i} k_{\alpha z} h}\right) \\
\Longleftrightarrow \\
\sum_{\beta} G_{\alpha \beta}^{\prime c} E_{\beta}^{\prime}=-Y_{\alpha}\left(C_{\alpha} e^{\mathrm{i} k_{\alpha z} h}-D_{\alpha} e^{-\mathrm{i} k_{\alpha z} h}\right)
\end{gathered}
$$

where:

$$
G_{\alpha \beta}^{\prime c}=\sum_{k_{\|} \sigma} \frac{Y_{k_{\|}}^{\prime}\left(1-R_{k_{\|} \sigma}\right)}{F_{k_{\|} \sigma}}\left\langle\alpha \mid k_{\|} \sigma\right\rangle\left\langle k_{\|} \sigma \mid \beta\right\rangle E_{\beta}^{\prime}
$$


Eventually, we have a similar set of equations to the one obtained in the case of the grid without cavity (eq. (34) and eq. 35). We have only to use $G^{\prime c}$ instead of $G^{\prime}$. Once the $E_{\alpha}$ and $E_{\alpha}^{\prime}$ are calculated by resolving the two-equation (if only one waveguide mode is taken into account) linear system, the electromagnetic fields can be derived in the structure and then for a given incident optical power, the power reflected by the whole structure and the amount of power absorbed in each layer can be easily calculated.

\subsection{Comparison with results obtained by Rigorous Coupled Wave Analysis}

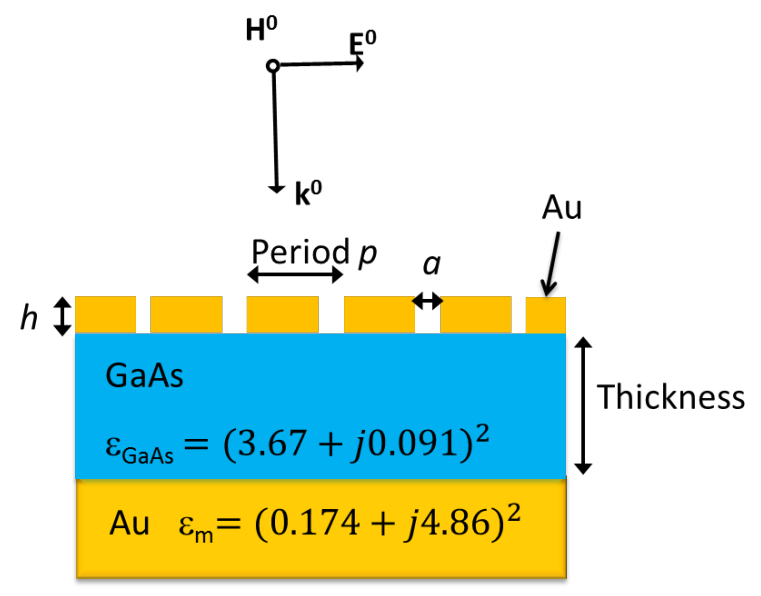

Figure 5: Resonant photoconductor using a $1 D$ array of slits as front mirror. $\lambda=$ $800 \mathrm{~nm}$.

The SMEF has been implemented to study the electromagnetic response to a normally incident plane wave (wavelength $\lambda=800 \mathrm{~nm}$ ) of a resonant photoconductor as shown in Figure 5 In Figure 6 are compared the absorbance of the GaAs layer as calculated by the SMEF and by a rigorous coupled wave analysis (RCWA)[13] as a function of the layer thickness and the period (the width of the slit $a=0.5 p$ ). The 400 simulation points required to plot the figures have needed a 13 times longer duration by using the RCWA method 


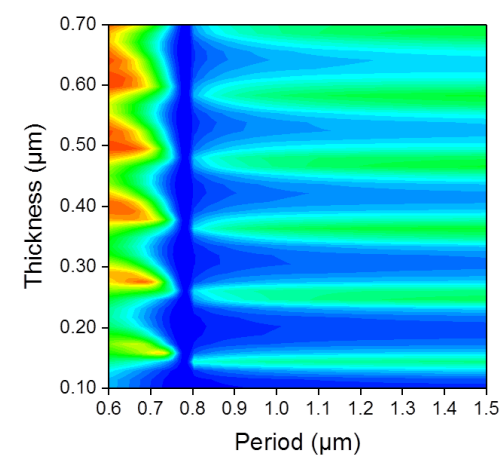

a)

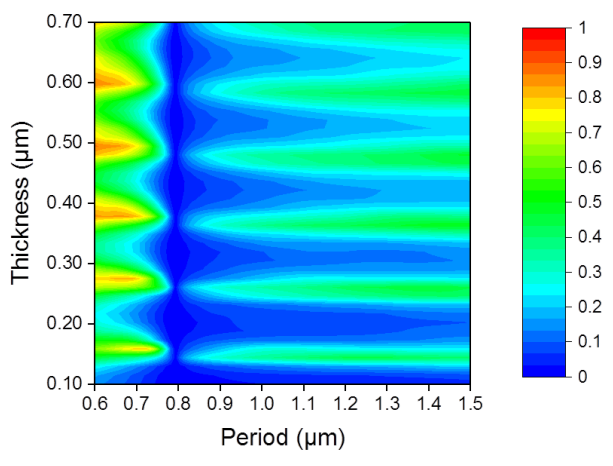

b)

Figure 6: Absorbance in the GaAs layer as a function of the thickness and the period of the array of slits wiyh $h=0.15 \mu \mathrm{m}$ and $a=0.5 \mathrm{p}$; a) calculated by the simplified modal expansion formalism, b) by the rigorous coupled wave analysis.

(despite the use of a small number of harmonics $n=20$ ), than by using the SMEF (number of waveguide mode $n_{s}=1$, number of diffracted mode $n_{D}=$ 1) implemented on the same platform (SCILAB). It is easily explained by the fact that each simulation point requires the inversion of a $2 \times 2$ matrix for the SMEF instead of a 20x20 matrix for the RCWA. It can be seen on Figure 6 that the numerical results obtained by both analysis are similar, nevertheless it can be noticed that the absorbance is overestimated by the SMEF. This is consistent with the fact that in we have considered here that the metal was a perfect metallic conductor (PEC) inside the slit in order to keep the very simple analytic form of the waveguide mode. Then the losses induced by the metal and the effective width of the slit are underestimated. As already mentioned before [12], the accuracy of this method could be improved by taking into account of the effect of the non perfect metal on the waveguide modes in the slits. 
${ }_{160}$

\section{Acknowledgement}

We gratefully acknowledge Mathias Vanwolleghem for fruitful discussions. This work is supported by the French Defense Agency DGA (Direction générale de l'armement), RENATECH (French Network of Major Technology Centers), Lille University, and the "Région Nord Pas de Calais Picardie".

\section{Appendix A. Calcul of the admittance $Y_{k \| \sigma}$}

Here, we demonstrate the relation between $E_{t}$ and $H_{t}$ in the s and p-polarization. In both case, it is based on the Maxwell-Faraday equation in CGS:

$$
\overrightarrow{r o t} \boldsymbol{E}=-\frac{1}{c} \frac{\partial \boldsymbol{B}}{\partial t}
$$

where $c$ is the speed of light in free space. Furthermore, if we assume that the materials have no magnetization, then $\boldsymbol{H}=\boldsymbol{B}$.

\section{1. s-polarization}

For a s-polarized incident plane wave, (i.e. $E_{z}=0, \phi(\boldsymbol{r}, t)=\boldsymbol{k} \cdot \boldsymbol{r}-\omega t$ ) of pulsation $\omega$ and of wave vector $\boldsymbol{k}=\left(k_{x}, k_{y}, k_{z}\right)^{t}$

$$
\overrightarrow{r o t} \boldsymbol{E}=-\frac{1}{c} \frac{\partial \boldsymbol{B}}{\partial t}
$$

becomes:

$$
\begin{array}{ccc}
j k_{x} \quad E_{x} & j \omega H_{x} \\
j k_{y} \times E_{y}=\frac{1}{c} \cdot & j \omega H_{y} \\
j k_{z} \quad 0 & j \omega H_{z} \\
& \Longleftrightarrow & \\
& \\
-k_{z} E_{y} & k_{\omega} H_{x} \\
k_{z} E_{x} & =k_{\omega} H_{y} \\
k_{x} E_{y}-j k_{y} E_{x} & k_{\omega} H_{z}
\end{array}
$$


Now, if we are only interested by the transverse parts of $\boldsymbol{E}$ and $\boldsymbol{H}$, we have indeed:

$$
\begin{array}{rcccc}
0 & H_{x} & H_{y} & E_{x} \\
\left|-\boldsymbol{u}_{\boldsymbol{z}} \times \boldsymbol{H}\right\rangle= & 0 & \times H_{y}= & -H_{x}=\boldsymbol{Y}_{\boldsymbol{k} \| \boldsymbol{s}} \cdot E_{y} \\
-1 & H_{z} & 0 & 0
\end{array}
$$

$170 \quad$ with $\boldsymbol{Y}_{\boldsymbol{k} \| \boldsymbol{s}}=\frac{k_{z}}{k_{\omega}}$.

\section{2. p-polarization}

For a p-polarized incident plane wave, (i.e. $H_{z}=0, \phi(\boldsymbol{r}, t)=\boldsymbol{k} \cdot \boldsymbol{r}-\omega t$ ) of pulsation $\omega$ and of wave vector $\boldsymbol{k}=\left(k_{x}, k_{y}, k_{z}\right)^{t}$

$$
\overrightarrow{r o t} \boldsymbol{E}=-\frac{1}{c} \frac{\partial \boldsymbol{B}}{\partial t}
$$

becomes:

$$
\begin{array}{ccc}
j k_{x} & E_{x} & j \omega H_{x} \\
j k_{y} \times & E_{y}=\frac{1}{c} \cdot & j \omega H_{y} \\
j k_{z} & E_{z} & 0 \\
& \Longleftrightarrow & \\
k_{y} E_{z}-k_{z} E_{y} & k_{\omega} H_{x} \\
k_{z} E_{x}-k_{x} E_{z}= & k_{\omega} H_{y} \\
k_{x} E_{y}-k_{y} E_{x} & 0
\end{array}
$$

So, it gives that $k_{x} E_{y}=k_{y} E_{x}$ and we know that without electric charge: $k_{x} E_{x}+$ $k_{y} E_{y}+k_{z} E_{z}=0$. It gives for the $x$ term:

$$
\begin{aligned}
k_{y} E_{z}-k_{z} E_{y} & =-\frac{k_{y} k_{x}}{k_{z}} E_{x}-\frac{k_{y}^{2}}{k_{z}} E_{y}-k_{z} E_{y} \\
& =-\frac{k_{y}^{2}}{k_{z}} E_{y}-\frac{k_{y}^{2}}{k_{z}} E_{y}-k_{z} E_{y} \\
& =-\frac{1}{k_{z}}\left(k_{\|}^{2}+k_{z}^{2}\right) E_{y} \\
& =-\frac{1}{k_{z}} \epsilon_{1} k_{\omega}^{2} E_{y}
\end{aligned}
$$


So for the $x$-term: $\boldsymbol{k}_{\boldsymbol{y}} \boldsymbol{E}_{\boldsymbol{z}}-\boldsymbol{k}_{\boldsymbol{z}} \boldsymbol{E}_{\boldsymbol{y}}=\boldsymbol{k}_{\boldsymbol{\omega}} \boldsymbol{H}_{\boldsymbol{x}} \Longleftrightarrow \frac{1}{\boldsymbol{k}_{z}} \boldsymbol{\epsilon}_{1} \boldsymbol{k}_{\boldsymbol{\omega}} \boldsymbol{E}_{\boldsymbol{y}}=-\boldsymbol{H}_{\boldsymbol{x}}$

175 Now, if we are interested by the $y$ term:

$$
\begin{aligned}
k_{z} E_{x}-k_{x} E_{z} & =\frac{k_{x} k_{y}}{k_{z}} E_{y}+\frac{k_{x}^{2}}{k_{z}} E_{x}+k_{z} E_{x} \\
& =\frac{k_{y}^{2}}{k_{z}} E_{x}+\frac{k_{x}^{2}}{k_{z}} E_{x}+k_{z} E_{x} \\
& =\frac{1}{k_{z}}\left(k_{\|}^{2}+k_{z}^{2}\right) E_{x} \\
& =-\frac{1}{k_{z}} \epsilon_{1} k_{\omega}^{2} E_{x}
\end{aligned}
$$

Eventually, for the $y$-term: $\boldsymbol{k}_{z} \boldsymbol{E}_{x}-\boldsymbol{k}_{x} \boldsymbol{E}_{z}=\boldsymbol{k}_{\boldsymbol{\omega}} \boldsymbol{H}_{\boldsymbol{y}} \Longleftrightarrow \frac{\boldsymbol{\epsilon}_{1} \boldsymbol{k}_{\boldsymbol{\omega}}}{\boldsymbol{k}_{z}} \boldsymbol{E}_{\boldsymbol{x}}=\boldsymbol{H}_{\boldsymbol{y}}$ Now, if we are only interested by the transverse parts of $\boldsymbol{E}$ and $\boldsymbol{H}$, we have indeed:

$$
\begin{array}{rlccr}
0 & H_{x} & H_{y} & E_{x} \\
\left|-\boldsymbol{u}_{\boldsymbol{z}} \times \boldsymbol{H}\right\rangle= & 0 & H_{y}= & -H_{x}=\boldsymbol{Y}_{\boldsymbol{k} \| \boldsymbol{p}} \cdot E_{y} \\
-1 & H_{z} & 0 & 0
\end{array}
$$

with $\boldsymbol{Y}_{\boldsymbol{k} \| \boldsymbol{p}}=\frac{k_{\omega} \epsilon_{1}}{k_{z}}$.

\section{References}

[1] M. Tonouchi, Cutting-edge terahertz technology, Nature Photonics 1 (2) (2007) 97-105. doi:10.1038/nphoton.2007.3.

URL http://dx.doi.org/10.1038/nphoton.2007.3

[2] J. Capmany, D. Novak, Microwave photonics combines two worlds, Nature Photonics 1 (6) (2007) 319-330. doi:10.1038/nphoton.2007.89.

URL http://dx.doi.org/10.1038/nphoton.2007.89

[ [3] T. Ishibashi, Y. Muramoto, T. Yoshimatsu, H. Ito, Unitraveling185 Carrier Photodiodes for Terahertz Applications, IEEE Journal of Selected Topics in Quantum Electronics 20 (6) (2014) 79-88. doi:10.1109/JSTQE.2014.2336537.

a URL http://ieeexplore.ieee.org/articleDetails.jsp?arnumber= 6861939 
口 [7] E. Peytavit, P. Latzel, F. Pavanello, G. Ducournau, J.-F. Lampin, CW

a Source Based on Photomixing With Output Power Reaching $1.8 \mathrm{~mW}$ at $250 \mathrm{GHz}$, IEEE Electron Device Letters 34 (10) (2013) 1277-1279. doi:10.1109/LED.2013.2277574.

URL http://ieeexplore.ieee.org/lpdocs/epic03/wrapper.htm? arnumber $=6588588$

[8] P. Latzel, F. Pavanello, E. Peytavit, M. Zaknoune, G. Ducournau, sources, in: M. Razeghi, E. Tournié, G. J. Brown (Eds.), Quantum Sensing and Nanophotonic Devices XII, International Society for Optics and Photonics, 2015, p. 937008. doi:10.1117/12.2080756

URL http://proceedings.spiedigitallibrary.org/proceeding. aspx?doi=10.1117/12.2080756 
„ [9] F. de León-Pérez, G. Brucoli, F. J. García-Vidal, L. Martín-Moreno, Theory

220 ㅁ on the scattering of light and surface plasmon polaritons by arrays of holes and dimples in a metal film, New Journal of Physics 10 (10) (2008) 105017. doi:10.1088/1367-2630/10/10/105017.

URL http://stacks.iop.org/1367-2630/10/i=10/a=105017

[10] F. J. Garcia-Vidal, L. Martin-Moreno, T. W. Ebbesen, L. Kuipers, Light passing through subwavelength apertures, Reviews of Modern Physics 82 (1) (2010) 729-787. doi:10.1103/RevModPhys .82.729.

URL http://link.aps.org/doi/10.1103/RevModPhys.82.729

[11] P. Bienstman, Rigorous and efficient modelling of wavelength scale photonic components, Ph.D. thesis, Gent University (2001).

230 [12] R. Gordon, A. G. Brolo, Increased cut-off wavelength for a subwavelength hole in a real metal, Optics Express 13 (6) (2005) 1933. doi:10.1364/OPEX.13.001933

a URL https://www.osapublishing.org/oe/abstract.cfm?uri= oe-13-6-1933

235 [13] M. G. Moharam, D. a. Pommet, E. B. Grann, T. K. Gaylord, Stable 1 implementation of the rigorous coupled-wave analysis for surface-relief gratings: enhanced transmittance matrix approach, Journal of the Optical Society of America A 12 (5) (1995) 1077. doi:10.1364/JOSAA.12.001077

URL http://www.opticsinfobase.org/abstract.cfm?URI=

${ }_{240}$ josaa-12-5-1077 